\title{
"in dem moment wo ich es dann erkenne dann ist es auch gleich wieder weg"1 Salienzeffekte in der Sprachperzeption
}

\author{
Christina A. Anders, Nicole Palliwoda, Saskia Schröder (Kiel)
}

\begin{abstract}
In context of the first study on folk linguistic concepts in the German language area carried out by the Kiel DFG research project "perceptual dialectology", this article looks at how salient features could be surveyed and categorized by a stimulus-response-test. After a definition of salience, the study design including the stimulus-response-test is presented. The test was created and modified during the project as a guessing game by the Institute for German Language (IDS Mannheim). The central question in this article is which linguistic features stimulate the informants to locate a speech sample on a map with predetermined cities and hence which salient features trigger the regional identification. In a second step, the speech samples are analyzed by the variables 'pleasantness' and 'correctness' defined by Dennis R. Preston. The central question here is: Are speech samples with a high pleasure value also automatically considered correct? Finally, an interpretation of metalinguistic comments in the speech examples will give more insight into folk linguistic concepts and the role of salient features in this regard.
\end{abstract}

\section{$1 \quad$ Einleitung}

Neuere Untersuchungen haben gezeigt, dass regionale Varietäten des Deutschen jeweils als unterschiedlich weit entfernt von der Standardvarietät eingestuft werden, auch wenn diese Varietäten objektiv gesehen dieselbe Anzahl an Abweichungen vom Standard aufweisen (cf. Kehrein 2012a, 2012b; Purschke 2012; Elmentaler/Gessinger/Wirrer 2010). So ist etwa das palatale /s/ in süddeutschen regionalen Varietäten offenkundig ein Trigger-Merkmal, das die regionale Zuordnung und Stereotypisierung auslöst (cf. Hundt 1992), während Merkmale wie die /r/-Vokalisierung in norddeutschen regionalen Varietäten als nicht-auffällige Variante der Standardsprache angesehen wird (cf. Elmentaler 2012). Zur Frage der Salienz sprachlicher Merkmale und ihrer Trigger-Funktion fehlten bislang im Bereich der Wahrnehmungsdialektologie noch weitgehend Daten. Diesen und weiteren Fragen widmet sich das laufende Kieler DFG-Forschungsprojekt Theoretische Modellierung und Erhebung laienlinguistischer Konzeptualisierungen zur deutschen Sprache in Deutschland, Österreich, der deutschsprachigen Schweiz, Liechtenstein, Luxemburg, Ostbelgien und Südtirol, in dessen Rahmen auch standardisierte Reiz-Reaktionstests durchgeführt werden. Dieser Beitrag gibt einen Einblick in die ersten Ergebnisse zur Salienz lautbezogener sowie außersprachlicher Merkmale mit Ausblick auf deren Triggerfunktion. Zu Beginn der Ausführungen soll auf den Salienz-Begriff eingegangen werden. Dieser wird seit einigen Jahren innerhalb der Dialektologie diskutiert. In einem zweiten Schritt werden kurz die Eckdaten des gesamten

\footnotetext{
1 Auszug aus Teiltranskript HB_TR_4551_36_12 des DFG-Projekts "Der deutsche Sprachraum aus der Sicht linguistischer Laien" (HU 661/4-1) der CĀ zu Kiel.
}

Linguistik online 66, 4/14 - http://dx.doi.org/ 10.13092/lo.66.1572

licensed under CC 3.0 
Projekts vorgestellt, wobei der Schwerpunkt das Setting des Ratespiels bildet ${ }^{2}$, bis dann im dritten Schritt erste Auswertungen hinsichtlich salienter Merkmale und metakommunikativer Daten dargestellt und interpretiert werden. ${ }^{3}$

\section{Schlaglichter zum Salienzbegriff 4}

In den ersten Arbeiten, in denen die Bedeutung des Begriffs Salienz eine Rolle spielt, spricht Schirmunski (1928/29 u. 1930) noch von Auffälligkeiten von sprachlichen Merkmalen. Diese klassifiziert er in primäre und sekundäre Merkmale, wobei primäre Merkmale leichter bzw. schneller abgebaut werden können und sekundäre länger erhalten bleiben (cf. Schirmunski 1928/29 u. 1930 sowie Lenz 2010). Trudgill (1986) verwendet als Erster den Begriff salience und betont dabei die subjektive Komponente der Bewertung sprachlicher Merkmale (cf. Trudgill 1986 sowie Lenz 2003 u. 2010). Nach Trudgill zeichnet sich salience durch die Merkmale "stigmatization, linguistic change, phonetic distance, and phonological contrast" aus (Trudgill 1986: 11). Auffällig ist nur das, was durch den Hörer bewertbar ist. Auer/Barden/Großkopf (1996: 163) teilen diese Auffassung und sehen in der Stigmatisierung von Dialektmerkmalen die Voraussetzung für Sprachwandel:

Die Auffälligkeit ('Salienz') von Dialektmerkmalen ist zwar eine notwendige Bedingung dafür, daß sie erlernt oder aufgegeben werden, sie muß sich jedoch, um zum Sprachwandel bzw. zur sprachlichen Anpassung zu führen, auf der attitudinalen Ebene mit einer entsprechend positiven oder negativen Besetzung des Merkmals paaren.

Für Mihm (1985: 184) ist hingegen die "Wirksamkeit expliziter Sprachnormvermittlung" ausschlaggebend für die Salienz von phonetischen Merkmalen. Salienz führt er zurück auf "Sprachrichtigkeitstopoi" (ibd.: 185).

Erste Tests zur phonetischen Auffälligkeit führen Herrgen/Schmidt (1985) durch Dialektalitätsbeurteilungen durch und etablieren in diesem Zusammenhang den Begriff der "Hörerurteil-Dialektalität" (ibd.: 23). Sie konstatieren, dass die

Beurteilung arealsprachlicher Merkmale als dialektal [...] unabhängig von der Systemrelevanz [ist, C.A.A.]. [...] Dialektalitätsbeurteilungen durch Hörer können nicht aus anderen Größen abgeleitet werden. Sie können weder aus dem Systemkontrast noch aus der Phonetik der Merkmale noch aus der arealen Verbreitung der Merkmale erklärt werden. Dialektalitätsbeurteilungen sind eine unabhängige Größe. (ibd.: 34-35)

Von Laien wahrgenommene arealsprachliche Merkmale gelten dann als salient, wenn sie im Prozess der "auf die Oralisierungsnorm gerichteten Makrosynchronisierungen" (Schmidt/Herrgen 2011: 62) als Bestandteile des intuitiven Wissens erworben werden. So auch Lenz, die Salienz als kognitive Auffälligkeit eines sprachlichen Merkmals versteht (cf. Lenz 2003 u. 2010). Das bedeutet, dass saliente Merkmale solche Merkmale sind, die aufgrund der subjektiven Wahrnehmung als Bestandteile des Sprachbewusstseins leichter und schneller zugänglich sind.

Auf die Kontextsensitivität weisen insbesondere Elmentaler/Gessinger/Wirrer hin. Ergebnisse aus dem SiN-Projekt ${ }^{5}$ zeigen, dass die subjektive Auffälligkeit von regionalen Merkmalen in

\footnotetext{
2 Details zu den einzelnen Projektbereichen können auf der Homepage nachgelesen werden: www.wahrnehmungsdialektologie.uni-kiel.de.

${ }^{3}$ Die im 4. Kapitel dargestellten Ergebnisse sind vorläufig, da die Erhebung erst Ende Januar 2013 abgeschlossen wurden.

${ }^{4}$ Ausführliche Überblicksdarstellungen und Diskussionen zum Salienzbegriff finden sich u. a. in Mattheier (1996), Kerswill/Williams (2002), Lenz (2010), Elmentaler/Gessinger/Wirrer (2010) und Purschke (2012).

${ }^{5}$ Ausführliche Informationen zu dem DFG-Projekt Sprachvariation in Norddeutschland können auf der folgenden Homepage nachgelesen werden: www.corpora.uni-hamburg.de/sin/index.html.
} 
direktem Zusammenhang mit dem Verwendungskontext steht (cf. Elmentaler/Gessinger/Wirrer 2010). Auch Purschke (2011: 109) kommt in seiner Dissertationsschrift zu dem Schluss, dass

für die Konzeption eines umfassenden Salienztests [...] neben der Beurteilung der perzeptiven Distinktheit (d.h. der Auffälligkeit) von Merkmalen auch Aspekte der interaktionalen Akzeptabilität (d.h. der Normhorizont) und die situative Signifikanz (d. h. die Interpretation der Situation) einzubeziehen sind.

Die Spezifizierung auf die situative Bedeutsamkeit führt Purschke konsequenterweise dazu, zwischen Salienz (auffällig/nicht auffällig) und Pertinenz (relevant/nicht relevant) als den "Basiskategorien sprachbezogener Hörerurteile" (ibd.: 308) zu differenzieren. Ausgehend vom Synchronisierungsmodell nach Schmidt/Herrgen (2011), in dem die Abläufe und Funktionen regionalsprachlicher Interaktionen im Mittelpunkt stehen, sieht Purschke (ibd.: 309) die

Beurteilung der Salienz sprachlicher Merkmale als Ergebnis individueller Perzeptionsprozesse, [...] Pertinenz hingegen als Resultat auf die Salienz bezogenen kognitiven Prozesse der Evaluation, die einem sprachlichen Merkmal zugeschrieben wird.

Im Rahmen des Kieler DFG-Projekts zur Wahrnehmung des deutschen Sprachraums aus der Sicht linguistischer Laien kann die Salienz von dialektkonzeptuellen Merkmalen nur im Hinblick auf die perzeptive Distinktheit untersucht werden. Damit wird im vorliegenden Beitrag Salienz als perzeptive Distinktheit verstanden. Eine auch situationssensitive und interaktional angelegte Untersuchung von Salienz, wie sie Purschke (2011) vorgelegt hat, wäre mit Sicherheit auch für wahrnehmungsdialektologische Untersuchungen sinnvoll, ist jedoch im Setting des Kieler Projekts nicht angelegt. Die anderen Teilprozesse von Hörerurteilen, die Purschke (2011) beschreibt, kommen innerhalb des gesamten Interviews teilweise zum Tragen, sie werden jedoch nicht in der Systematik durchgeführt. Somit liefert das Datenmaterial aber auch Ergebnisse zu anderen Aspekten wie beispielsweise der Pertinenz von salienten Merkmalen.

\section{$3 \quad$ Eckdaten des Kieler DFG-Projekts "Wahrnehmungsdialektologie"}

Gegenstand des Projekts sind die linguistischen, geografischen, sozialen, kognitiven und visualisierten Raumkonzeptionen von regionalen Varietäten des Deutschen aus der Sicht deutschsprachiger linguistischer Laien in Deutschland, Österreich, der deutschsprachigen Schweiz, Liechtenstein, Luxemburg, Ostbelgien und Südtirol. Dabei werden folgende Fragestellungen verfolgt:

1. Welche sprachlichen und nichtsprachlichen Merkmale assoziieren und perzipieren die Gewährspersonen mit den von ihnen repräsentierten Dialektkonzepten?

2. Wie sehen die arealen Strukturen dieser Dialektkonzepte (mental maps) aus?

3. Wie verhält es sich mit der evaluativen Struktur der Dialektkonzepte (Auto- und Heterostereotype)?

4. Welche Normkonzepte bestehen bei den Gewährspersonen und wie verhalten sich diese hinsichtlich der Salienz?

5. Welche Konsequenzen können aus den Ergebnissen für den Sprachwandel gewonnen werden?

Mit den Fragen 1 und 4 werden wahrgenommene sprachliche Merkmale sowie deren laienlinguistische Kategorisierung systematisch erfasst (Frage 1) und deren perzeptive Distinktheit ermittelt (Frage 4).

Die Erhebungsphase lief von Februar 2012 bis Januar 2013, im Anschluss daran werden die Daten ausgewertet. Alle Daten wurden in direkten teilstrukturierten Leitfadeninterviews 
erhoben, vollständig aufgezeichnet und orthografisch transkribiert. Dabei wurden im Idealfall sechs Gewährspersonen pro Ort aus drei verschiedenen Altersgruppen befragt. ${ }^{6}$

Die Grundlage des Offline-Ratespiels bildet ein Online-Ratespiel des Deutsch heute-Projekts 7 vom Institut für Deutsche Sprache (Mannheim) "Hör mal wo der spricht"8, das leicht modifiziert wurde. Den Gewährspersonen wurden dabei neun Sprechproben vorgespielt, die so schnell wie möglich zur Auswahl stehenden Städten, die auf einer Karte eingeblendet sind, zugeordnet wurden (siehe Abb.1).

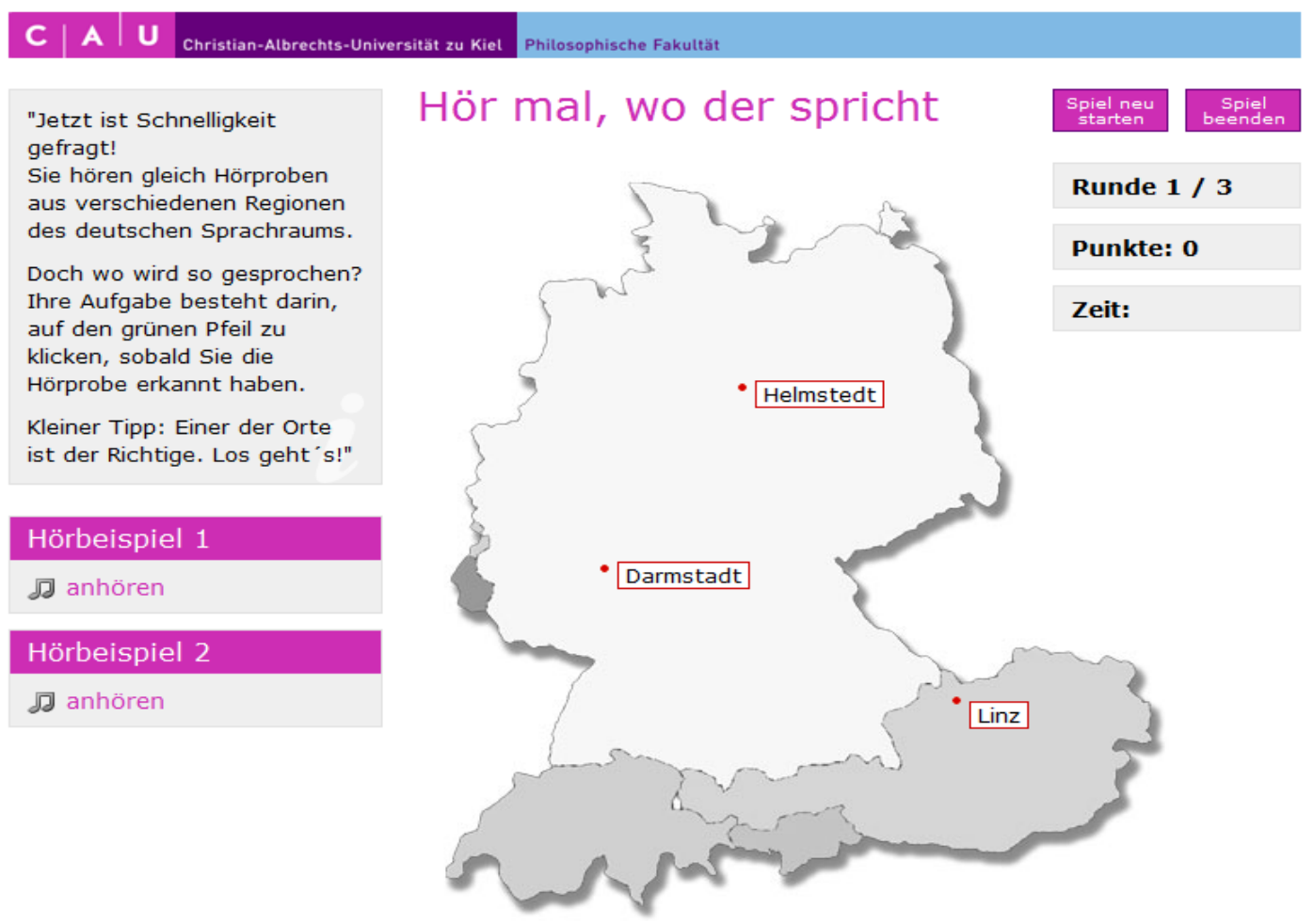

Abbildung 1: Screenshot des Ratespiels (in Kooperation mit dem IDS Mannheim).

Das Spiel besteht aus drei Runden, wobei in der ersten Runde zwei Sprechproben zu hören sind und drei Orte zur Auswahl stehen, in der zweiten Runde drei Sprechproben und vier Orte und in der dritten Runde vier Sprechproben und fünf Auswahlorte. Die Sprachlagen der verwendeten Sprechproben ${ }^{9}$ sind auf den Ebenen Regionalakzent bzw. mittlerer Substandard zu verorten. Die Frage, inwiefern nationale Varietäten übergreifende Gefallens- und Normurteile die Zuordnung und Bewertung der Samples beeinflussten, wird in diesem Beitrag nicht gesondert behandelt. In jedem Sample wird der Text Nordwind und Sonne von jeweils einem männlichen Sprecher vorgelesen. ${ }^{10}$

\footnotetext{
${ }^{6}$ Dabei wird pro Erhebungsort ein Schüler zwischen 16 und 20 Jahren (geboren bis 1996) befragt (Altersgruppe I), zwei Lehrer zwischen 30 und 50 Jahren (geboren bis 1982) und drei Lehrer zwischen 55 und 65 Jahren (geboren bis 1957).

${ }^{7}$ Weitere Informationen zu diesem Projekt können auf der folgenden Homepage nachgelesen werden: www.idsmannheim.de/prag/AusVar/Deutsch_heute.

${ }^{8}$ Auf der folgenden Homepage kann gern am Online-Ratespiel des Deutsch heute-Projekts teilgenommen werden: http://multimedia.ids-mannheim.de/hoermal/web.

${ }^{9}$ Die Wahl der Sprechproben bezog sich auf die zehn meist genannten Dialektbezeichnungen der Probanden aus der Pilotstudie (cf. Hundt 2010: 179-219).

${ }^{10}$ Sechs Sprechproben stammen aus dem REDE-Projekt und drei wurden selbst aufgenommen.
} 


\begin{tabular}{|l|l|l|l|}
\hline Dialektverband & Belegort & D-Wert $^{11}$ & Quelle \\
\hline Zentralhessisch/Rheinfränkisch & Darmstadt & 0,9 & REDE \\
\hline Obersächsisch & Dresden & 0,8 & REDE \\
\hline Hochalemannisch & Zürich & 1,1 & Uni Zürich \\
\hline Mittelbairisch & Wien & 0,9 & Uni Wien \\
\hline Brandenburgisch & Potsdam & 0,5 & REDE \\
\hline Nordniederdeutsch & Rendsburg & 0,2 & REDE \\
\hline Mittelbairisch & Traunstein & 0,9 & REDE \\
\hline Schwäbisch & Ulm & 0,9 & REDE \\
\hline $\begin{array}{l}\text { Standardsprachliches } \\
\text { Kontrollsample }\end{array}$ & Helmstedt & 0,5 & CAU Kiel \\
\hline
\end{tabular}

Tabelle 1: Sprechprobenauswahl und D-Werte.

Alle Sprechproben werden in randomisierter Reihenfolge vorgespielt. Eine Ausnahme bildet das standardsprachliche Kontrollsample, das stets als erstes zu hören ist und im Interview offiziell als Testrunde angekündigt wird. Damit soll geprüft werden, ob das Sample als Standardvarietät identifiziert wird. Falls ja, dann wäre davon auszugehen, dass diese Tonprobe im Interview von den Gewährspersonen als greifbare d. h. aktuelle Referenz genutzt werden kann, wenn die anderen Sprechproben mit dem Standardkonzept verglichen werden sollen. Die Ergebnisse zu diesem Sample werden in der weiteren Auswertung zur perzeptiven Distinktheit ausgeklammert.

Der für das Standarddeutsche eigentlich zu hohe D-Wert dieses Samples wirkte sich nicht negativ auf die intendierte Kontrollfunktion aus. Von insgesamt 42 Befragten waren immerhin 30 der Meinung, dass es sich bei diesem Sample um eine "hochdeutsche" 12 Aussprache handelt (cf. Tab. 2).

\begin{tabular}{|l|l|l|}
\hline $\mathbf{N}=\mathbf{4 2}$ & + Hochdeutsch & - Hochdeutsch \\
\hline Kontrollsample & 30 & 12 \\
\hline
\end{tabular}

Tabelle 2: Benennung des Kontrollsamples durch die GPs.

Grundlage der für die Zuordnung der Sprechproben zur Auswahl stehenden Städte bilden 61 Orte, die in einem Vortest ermittelt wurden. Ziel dieses Vortests war es herauszufinden, welche Städte im Erhebungsraum am bekanntesten sind. Für die Ermittlung dieser kognitiv relevanten Ortspunkte wurde ein Online-Test entwickelt, der von Mai bis September 2011 freigeschalten war und an dem insgesamt 653 Personen teilnahmen. Den Teilnehmenden wurde zunächst eine Karte mit den äußeren Umrissen des Erhebungsraums eingeblendet, im Anschluss daran sollten in eine entsprechende Maske alle Städte, die den Teilnehmenden beim Anblick der Karte spontan einfielen, notiert werden. Die 61 am häufigsten genannten Städte bilden das Sample für die Makrokartierung (Frage 3, s. o.) und das Ratespiel, wobei für das Ratespiel noch die Herkunftsorte der Sprecher ergänzt wurden. Sowohl die Sprechproben als auch die Städte

11 Die Ermittlung der D-Werte erfolgte durch Saskia Schröder und durch das REDE-Projekt (www.regionalsprache.de).

12 Das ist die Bezeichnung, die die Interviewpartner dem Kontrollsample gegeben haben, als sie das Sprachbeispiel hörten. 
wurden in randomisierter Reihenfolge zur Auswahl gestellt. Zusätzlich wurde die Zeit, die jede Gewährsperson für die Zuordnung benötigte, erhoben. ${ }^{13}$ Bei der Umsetzung kam es leider zu technischen Problemen, sodass die Zeitmessung aufgrund von Verzögerungen bei einigen Durchgängen teilweise keine exakten Daten liefern konnte. Da die Gewährspersonen einige Sprechproben aber bereits identifizieren und kommentieren, noch während das Hörbeispiel lief (on-time), sollten die Zeiten und Zuordnungen im Rahmen der weiteren Auswertung entsprechend nachgeprüft werden. Im Anschluss daran wurden die Gewährspersonen gebeten $\mathrm{zu}$ begründen, warum sie das Sample einem Auswahlort zugeordnet hatten. Die Interviewpersonen sollten außerdem mithilfe einer Ratingskala beschreiben, wie ihnen die Sprechproben gefallen haben und anschließend beurteilen, wie korrekt sie die Sprachbeispiele einschätzen würden. Korrektheit meint in diesem Zusammenhang, wie nah bzw. entfernt vom intendierten Standardkonzept die Gewährsperson die Sprechprobe einordnen würde. ${ }^{14} \mathrm{Im}$ Laufe der Erhebungen ergaben sich teilweise auch hier Schwierigkeiten, weil einige Gewährspersonen ihre Unsicherheit darüber signalisierten, was unter "Korrektheit" zu verstehen sei: "gutes Deutsch", "grammatisch[e] [K]orrekt[heit]" oder "gutes, d. h. korrektes regionales Deutsch"? Diese Unklarheiten konnten jedoch schon im weiteren Verlauf des Interviews durch die Exploratorinnen behoben werden.

\section{Werkstattbericht: Erste Ergebnisse}

Die Grundlage für die hier vorliegenden Ergebnisse bildet eine Stichprobe von 42 befragten Personen aller Altersgruppen aus den Erhebungsorten Eppingen, Hameln, Neuruppin, Schleiden, Simmern/Hunsrück, Vaduz/L., Coburg und Meran (siehe Tab. 3).

\begin{tabular}{|l|l|l|l|}
\hline Altersgruppen & $\mathbf{1 6 - 2 0}$ J. & $\mathbf{3 0 - 5 0}$ J. & $\mathbf{5 5 - 6 5}$ J. \\
\hline Anzahl & 11 & 15 & 16 \\
\hline
\end{tabular}

Tabelle 3: Stichprobe der hier präsentierten Daten $(N=42)$.

Mit Blick auf die vorliegenden Daten interessieren drei Fragestellungen:

1. Welche sprachlichen und nichtsprachlichen Merkmale werden von den Gewährspersonen in den Sprechproben perzipiert?

2. Welche lautlichen Merkmale sind salient?

3. Welche salienten lautlichen Merkmale triggern die regionale Identifikation?

Die erhobenen perzipierten Merkmale werden nach dem von Anders (2010: 269) erstellten Kategoriensystem klassifiziert. Im Folgenden soll ein erster kleiner Einblick in die Auswertung von salienten lautlichen sowie nichtsprachlichen Merkmalen und ihr Triggerpotenzial gegeben werden. Mit dem für wahrnehmungsdialektologische Untersuchungen geeigneten Kategoriensystem sollen die laienlinguistischen Beschreibungen möglichst authentisch abbildbar systematisiert werden, sodass auch unspezifische allgemeine Beschreibungen wie z. B. "die betonung der vokale ist so komisch"15 als perzipierte Merkmale klassifizierbar sind. Ganz bewusst wurde darauf verzichtet, die laienlinguistischen Beschreibungen in ein systemlinguistisches Kategoriensystem "einzupassen".

Bevor auf die Frage eingegangen wird, welche sprachlichen und nichtsprachlichen Merkmale von den Interviewpersonen mit den Sprechproben perzipiert wurden, soll kurz auf die Auswertung der Ergebnisse, inwiefern die Sprechproben der richtigen Stadt zugeordnet

\footnotetext{
${ }^{13}$ Die Zeitmessung beginnt beim ersten Klick auf den Button Anhören und endet beim Klick auf die entsprechende Stadt.

${ }^{14}$ Vgl. Preston 1999: xxiii-xl: "grade of correctness".

${ }^{15}$ Aussage einer GP innerhalb des Interviews zu einer Sprechprobe.
} 
wurden, wie sie den Interviewten gefallen haben und als wie "korrekt" die Sprachbeispiele eingeschätzt wurden, eingegangen werden. Die folgende Tabelle stellt dar, welche der Samples von wie vielen Informanten richtig lokalisiert wurden.

\begin{tabular}{|l|l|l|}
\hline Sample & falsch & richtig \\
\hline Mittelbairisch (Traunstein) & 15 & 27 \\
\hline Brandenburgisch (Potsdam) & 24 & 18 \\
\hline Zentralhessisch/Rheinfränkisch (Darmstadt) & 23 & 19 \\
\hline Hochalemannisch (Zürich) & 2 & 40 \\
\hline Mittelbairisch (Wien) & 15 & 27 \\
\hline Nordniederdeutsch (Rendsburg) & 14 & 28 \\
\hline Obersächsisch (Dresden) & 6 & 36 \\
\hline Schwäbisch (Ulm) & 11 & 31 \\
\hline Standardsprachliches Kontrollsample (Helmstedt) & 17 & 25 \\
\hline
\end{tabular}

Tabelle 4: Zuordnung der Sprechproben $(N=42)$.

Insgesamt wird deutlich, dass die Sprechproben mehrheitlich korrekt zugeordnet wurden. Ausnahmen bilden die brandenburgische und zentralhessische Sprechprobe. Obwohl die Auswahlstädte in zufälliger Reihenfolge aufgezeigt werden, ist nicht auszuschließen, dass die Städte, die im Ratespiel jeweils zur Auswahl standen, die Zuordnung beeinflusst haben. So kann es sein, dass die Zuordnung eines Sprachbeispiels nach dem Ausschlussprinzip vorgenommen wurde. Für eine präzise Auswertung von Einflussfaktoren bei der lokalen Identifikation könnte zukünftig mit erhoben werden, welche Städte jeweils zur Auswahl standen.

Die nächste Tabelle bildet ab, wie vielen Befragten die jeweiligen Sprechproben auf einer Skala von -3 (gar nicht) bis +3 (sehr gut) gefallen bzw. nicht gefallen haben. 


\begin{tabular}{|c|c|c|c|c|c|c|c|c|c|}
\hline \multicolumn{8}{|c|}{$\begin{array}{l}\text { "Wie gefällt Ihnen diese Sprechweise?" } \\
\text { (auf einer Skala von }-3 \text { gar nicht bis }+3 \text { sehr gut) }\end{array}$} & \multirow[t]{2}{*}{ Mittelwert } & \multirow[t]{2}{*}{$\begin{array}{l}\text { Standard- } \\
\text { abweichung }\end{array}$} \\
\hline Sample & -3 & -2 & -1 & 0 & 1 & 2 & 3 & & \\
\hline Mittelbairisch (Traunstein) & 0 & 2 & 5 & 7 & 7 & 14 & 7 & 1,12 & 1,452 \\
\hline $\begin{array}{l}\text { Brandenburgisch } \\
\text { (Potsdam) }\end{array}$ & 0 & 2 & 2 & 4 & 9 & 19 & 6 & 1,40 & 1,270 \\
\hline $\begin{array}{l}\text { Zentralhessisch/Rheinfränk } \\
\text { isch (Darmstadt) }\end{array}$ & 1 & 5 & 7 & 8 & 9 & 10 & 2 & 0,36 & 1,543 \\
\hline Hochalemannisch (Zürich) & 0 & 3 & 3 & 10 & 9 & 12 & 5 & 0,93 & 1,404 \\
\hline Mittelbairisch (Wien) & 0 & 3 & 5 & 4 & 8 & 17 & 5 & 1,10 & 1,462 \\
\hline $\begin{array}{l}\text { Nordniederdeutsch } \\
\text { (Rendsburg) }\end{array}$ & 0 & 2 & 4 & 5 & 8 & 20 & 3 & 1,17 & 1,305 \\
\hline Obersächsisch (Dresden) & 4 & 6 & 10 & 7 & 5 & 7 & 3 & $-0,14$ & 1,775 \\
\hline Schwäbisch (Ulm) & 1 & 0 & 8 & 10 & 12 & 8 & 3 & 0,62 & 1,324 \\
\hline $\begin{array}{l}\text { Standardsprachliches } \\
\text { Kontrollsample } \\
\text { (Helmstedt) }\end{array}$ & 0 & 0 & 1 & 4 & 5 & 16 & 16 & 2,00 & 1,059 \\
\hline
\end{tabular}

Tabelle 5: Gefallen der Sprechproben $(\mathrm{N}=42)$.

Auffällig ist, dass die Sprechproben überwiegend positiv eingeschätzt werden. Die Ausnahme bildet das obersächsische Beispiel: Hier liegen die meisten Gefallen-Werte im Negativbereich. Mit Blick auf die positiven Gefallen-Werte lässt sich erkennen, dass das Kontrollsample den meisten Personen (37) gefällt, dicht gefolgt von der brandenburgischen (34) und nordniederdeutschen Sprechprobe (31). Schlusslichter im Bereich der positiven Bewertung bilden mit 21 Personen die Sprechprobe aus Darmstadt und das obersächsische Sprachbeispiel (15). Unterstrichen werden die Ergebnisse auch durch die Mittelwerte sowie die Streuung der Werte. Das Kontrollsample hat im Mittel die durchweg positivsten Einschätzungen $(2,00)$ erhalten und dies auch mit der geringsten Standardabweichung $(1,059)$. Dahingegen zeigt der Mittelwert des obersächsischen Beispiels die negativste Bewertung $(-0,14)$, wobei hier die höchste Standardabweichung (1,775) auftritt. Hinsichtlich der Bewertung der einzelnen Samples sind die Ergebnisse nicht neu und bestätigen vorhergehende Untersuchungen (cf. u. a. Hundt 1992 u. 2010, Allensbacher Berichte 2008).

Ein anderes Bild bietet hingegen die Tabelle zu den Korrektheits-Werten (cf. Tab. 6). Korrektheit meint in diesem Zusammenhang, als wie nah bzw. fern vom intendierten Standardkonzept die Gewährsperson die Sprechprobe einordnen würde. Bei dieser Frage wurde auf die erste Sprechprobe als Vergleichsoption verwiesen, da dieses Beispiel bei allen als erstes eingespielt wurde und als Standardsample gelten sollte. 


\begin{tabular}{|l|l|l|l|l|l|l|l|l|l|}
\hline $\begin{array}{l}\mid 7 \\
\text { "Wie sprachlich korrekt/richtig haben Sie diese }\end{array}$ & \multicolumn{1}{|l|}{$\begin{array}{l}\text { Mittel- } \\
\text { wert } \\
\text { (auf einer Skala von -3 absolut inkorrekt bis +3 sehr } \\
\text { korrekt) }\end{array}$} & $\begin{array}{l}\text { Standard- } \\
\text { abweichung }\end{array}$ & \\
\hline Sample & -3 & -2 & -1 & 0 & 1 & 2 & 3 & & 1,468 \\
\hline Mittelbairisch (Traunstein) & 0 & 2 & 11 & 8 & 9 & 7 & 5 & 0,55 & 1,034 \\
\hline Brandenburgisch (Potsdam) & 0 & 1 & 1 & 1 & 7 & 23 & 9 & 1,83 & 1,495 \\
\hline $\begin{array}{l}\text { Zentralhessisch/Rheinfränk } \\
\text { isch (Darmstadt) }\end{array}$ & 2 & 5 & 6 & 6 & 14 & 9 & 0 & 0,24 & 1,443 \\
\hline Hochalemannisch (Zürich) & 0 & 3 & 13 & 7 & 7 & 10 & 2 & 0,33 & 1,464 \\
\hline Mittelbairisch (Wien) & 0 & 3 & 8 & 9 & 8 & 10 & 4 & 0,62 & 1,087 \\
\hline $\begin{array}{l}\text { Nordniederdeutsch } \\
\text { (Rendsburg) }\end{array}$ & 0 & 0 & 3 & 1 & 6 & 20 & 12 & 1,88 & 1,554 \\
\hline Obersächsisch (Dresden) & 1 & 5 & 8 & 8 & 8 & 10 & 2 & 0,31 & 1,330 \\
\hline Schwäbisch (Ulm) & 0 & 4 & 5 & 12 & 9 & 11 & 1 & 0,50 & 0,547 \\
\hline $\begin{array}{l}\text { Standardsprachliches } \\
\text { Kontrollsample } \\
\text { (Helmstedt) }\end{array}$ & & & & & & & & 2,57 & \\
\hline
\end{tabular}

Tabelle 6: Korrektheit der Sprechproben $(\mathrm{N}=42)$.

Zum einen bestätigen die Ergebnisse die Aussage, dass es sich bei der Helmstedter Sprechprobe überwiegend um eine wahrgenommene standardsprachliche Variante handelt, da 42 Personen dieses Beispiel als korrekt bis sehr korrekt einschätzen (cf. ebenfalls Tab. 2). Bei diesem Sprachbeispiel lassen sich keine negativen Werte finden. Dies bestätigen ebenfalls der Mittelwert $(2,57)$ sowie die geringste Standardabweichung $(0,547)$. Zum anderen lassen sich hier erste Verbindungen zwischen den Gefallen- und Korrektheits-Werten ablesen: Die Sprechproben aus Potsdam und Rendsburg erhalten - neben dem Kontrollsample - sowohl beim Gefallen als auch bei der Korrektheit die meisten positiven Werte ([34/39]/[31/38]), was gleichfalls durch die Mittelwerte der jeweiligen Sprachbeispiele bestätigt werden kann. Obwohl die obersächsische Sprechprobe die geringsten Gefallen-Werte erhalten hat, ist sie bei der Korrektheit relativ gleich zu der Darmstädter und Zürcher Probe (cf. Mittelwerte Tab. 7). Auffällig ist aber, dass die Zürcher Sprechprobe die meisten negativen Werte (16, Mittelwert 0,33) bekommen hat, obwohl mehr als die Hälfte der Probanden (26) beim Gefallen positive Aussagen getroffen haben. Damit scheint es saliente Merkmale zu geben, die ein positives Konzept des Schweizerdeutschen triggern, im vorliegenden Fall durch die Zürcher Sprechprobe. Diese Merkmale werden aber bewusst auch als inkorrekt wahrgenommen. Solche Auffälligkeiten scheint es ebenfalls bei den mittelbairischen Sprechproben zu geben (cf. Mittelwert Tab. 5 und 6). Ebenfalls scheint die Streuung der Ergebnisse bei denjenigen Sprechproben besonders hoch zu sein, die im Mittel eher als weniger korrekt eingeschätzt werden (cf. Standardabweichung Tab. 6).

Es lässt sich - wie in anderen Untersuchungen - feststellen, dass bestimmte Sprachbeispiele polarisieren, es aber auch Sprechproben gibt, die sowohl als angenehm als auch als korrekt empfunden werden. Aufgrund dessen wurde für die Gefallen- und Korrektheits-Werte eine Kreuztabelle erstellt, um herauszufinden, welche Gefallen-Werte wie häufig und mit welchen Korrektheits-Werten auftreten (cf. Tab. 7). 


\begin{tabular}{|c|c|c|c|c|c|c|c|c|}
\hline & & \multicolumn{7}{|c|}{$\begin{array}{l}\text { "Wie sprachlich korrekt/richtig haben Sie diese } \\
\text { Sprechweise empfunden?" } \\
\text { (auf einer Skala von }-3 \text { absolut inkorrekt bis }+3 \text { sehr } \\
\text { korrekt) }\end{array}$} \\
\hline & & -3 & -2 & -1 & $\mathbf{0}$ & $\mathbf{1}$ & 2 & 3 \\
\hline \multirow{7}{*}{$\begin{array}{l}\text { Wie gefällt Ihnen } \\
\text { diese Sprechweise } \\
\text { (auf einer Skala }-3 \\
\text { gar nicht bis }+3 \\
\text { sehr gut)? }\end{array}$} & -3 & 2 & 2 & 1 & 1 & 0 & 0 & 0 \\
\hline & -2 & 1 & 8 & 8 & 2 & 2 & 2 & 0 \\
\hline & -1 & 0 & 7 & 15 & 10 & 5 & 6 & 2 \\
\hline & $\mathbf{0}$ & 0 & 2 & 12 & 15 & 6 & 15 & 9 \\
\hline & 1 & 0 & 1 & 7 & 11 & 23 & 20 & 10 \\
\hline & 2 & 0 & 3 & 10 & 10 & 24 & 58 & 18 \\
\hline & 3 & 0 & 0 & 2 & 3 & 9 & 15 & 21 \\
\hline
\end{tabular}

Tabelle 7: Kreuztabelle der Gefallen- und Korrektheitswerte $(N=42)$.

Es wird deutlich, dass die meisten positiven Gefallen- mit den meisten positiven KorrektheitsWerten auftreten. Ein Blick in das folgende Diagramm bestätigt diese Aussage:

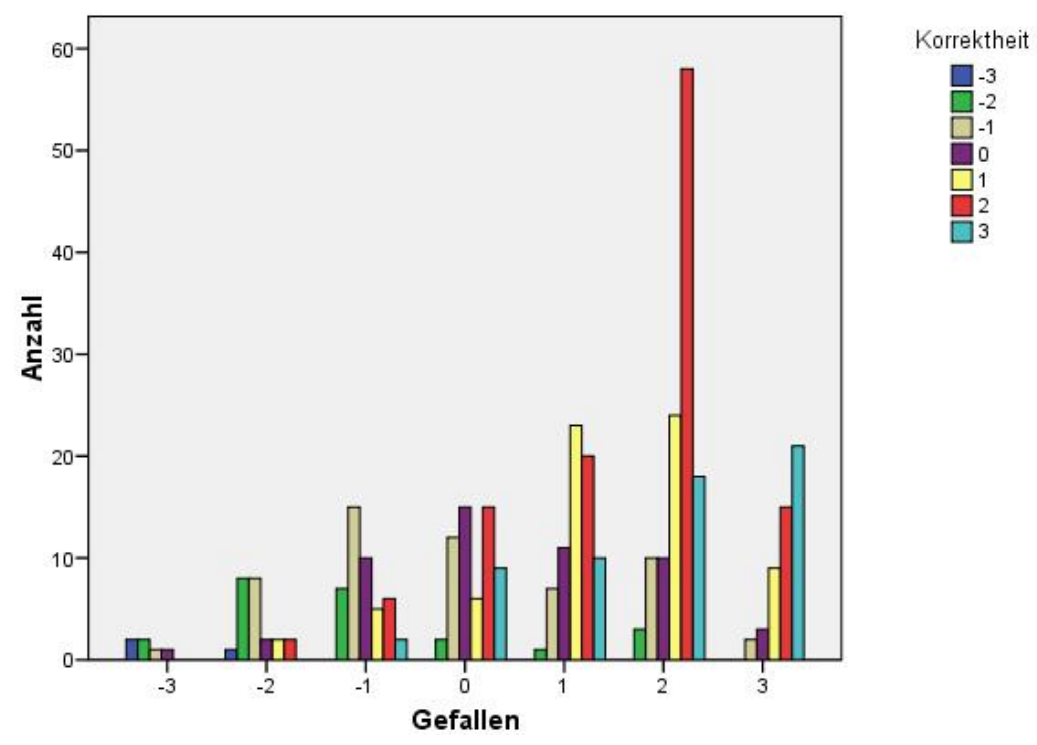

Abbildung 2: Balkendiagramm der Gefallen- und Korrektheitswerte aus der Kreuztabelle.

Die grafische Darstellung veranschaulicht sehr gut, dass einerseits Sprechproben, die den Probanden gefallen haben, auch meistens als korrekt angesehen wurden. Andererseits zeigt die Abbildung, dass Sprachbeispiele, die den Probanden nicht gefallen haben, eher kaum bis nie als korrekt eingestuft wurden. Somit lässt sich aus der Kreuztabelle und dem Diagramm ein Zusammenhang zwischen Gefallen und Korrektheit erkennen, der mit Hilfe des Korrelationstests ${ }^{16}$ bestätigt werden kann (cf. Tab. 8).

16 Korrelation nach Pearson, intervallskalierte Daten. 


\begin{tabular}{|l|l|l|l|}
\hline & & Gefallen & Korrektheit \\
\hline Gefallen & $\begin{array}{l}\text { Korrelation nach } \\
\text { Pearson }\end{array}$ & 1 &, $537(* *)$ \\
\hline & Signifikanz (2-seitig) & &, 000 \\
\hline & $\mathrm{N}$ & 378 & 378 \\
\hline $\begin{array}{l}\text { Korrekth } \\
\text { eit }\end{array}$ & $\begin{array}{l}\text { Korrelation nach } \\
\text { Pearson }\end{array}$ &, $537(* *)$ & 1 \\
\hline & Signifikanz (2-seitig) &, 000 & \\
\hline & $\mathrm{N}$ & 378 & 378 \\
\hline
\end{tabular}

Tabelle 8: Korrelation zw. Gefallen und Korrektheit der 42 Probanden.

Im nächsten Schritt soll nun auf die sprachlichen Merkmale eingegangen werden, die die Interviewpersonen perzipiert und nach deren Kriterien sie die Zuordnung der Sprechproben vorgenommen haben.

Die Merkmalsklasse "Lautliche Besonderheiten" setzt sich aus insgesamt vier Untergruppen zusammen, die jeweils weiter nach linguistischen Kriterien differenziert werden (cf. Anders 2010: 269):

\section{Lautliche Besonderheiten (auditiv-phonetische Beschreibungen)}

(11) Vokalische Assoziation

(111) Unspezifische allgemeine Beschreibungen

(112) Spezifische allgemeine Beschreibungen

(113) Vokalfrequenzen

(114) Vokalqualitäten

(12) Konsonantische Assoziation

(121) Unspezifische allgemeine Beschreibungen

(122) Spezifische allgemeine Beschreibungen

(123) Konsonantenqualitäten

(13) Prosodische Assoziation

(131) Quantität

(132) Intonation

(133) Akzent

(14) Artikulatorische Assoziation

(141) Stil

(142) Aussprache

Insgesamt haben sich die Befragten bei ihren Begründungen, woran sie die Herkunft des Sprechers erkannt haben, stark zurückgehalten. Häufig lokalisierten sie die Samples "intuitiv aus dem Bauch heraus" oder nach dem "Ausschlussprinzip". Eher selten wurden konkrete sprachliche Merkmale benannt und nur in Einzelfällen wurden diese mit Bewertungen kombiniert.

Die in dieser Stichprobe insgesamt 285 Nennungen von perzipierten lautlichen Merkmalen verteilen sich auf die vier Untergruppen wie folgt: 


\begin{tabular}{|l|l|l|l|l|}
\hline Merkmalskategorien & $\mathbf{n}>\mathbf{0}$ & $\mathbf{n}>\mathbf{1}$ & $\mathbf{n}>\mathbf{3}$ & $\varnothing$ je Inf. (n > 0 \\
\hline Konsonantische Assoziation & 101 & 84 & 73 & 2,4 \\
\hline Vokalische Assoziation & 89 & 62 & 23 & 2,1 \\
\hline Prosodische Assoziation & 54 & 49 & 40 & 1,3 \\
\hline Artikulatorische Assoziation & 41 & 32 & 20 & 0,9 \\
\hline$\sum$ & 285 & 227 & 156 & 6,8 \\
\hline
\end{tabular}

Tabelle 9: Anzahl der von den 42 Befragten genannten perzipierten Merkmale der Kategorie "Lautliche Besonderheiten"

Unter Berücksichtigung der Einzelnennungen benennt dabei jeder Informant durchschnittlich 6,8 Merkmale, ohne die Einzelnennungen sind es immerhin noch 5,4 Einzelmerkmale. Diese verteilen sich jedoch sehr ungleichmäßig auf die vier Subgruppen. Für die befragten linguistischen Laien scheinen konsonantische Merkmale in den Samples mit Abstand am auffälligsten zu sein. Von den insgesamt 101 auffälligen Merkmalen aus dem konsonantischen Bereich werden immerhin 84 mehr als ein Mal und $73 \mathrm{mehr}$ als drei Mal genannt. Dagegen fallen Merkmale der artikulatorischen Assoziation deutlich weniger auf, durchschnittlich etwa nur ein Merkmal pro Informant unter Berücksichtigung der Einzelnennungen. Der Vergleich der vokalischen mit den prosodischen Merkmalen zeigt eine diametrale Verteilung der Nennungen. Während das Spektrum der vokalisch perzipierten Merkmale deutlich vielseitiger ist als bei den Merkmalen der Prosodie und die Anzahl der Einzelnennungen entsprechend größer ausfällt, werden wenige prosodische Merkmale von mehr Informanten perzipiert. Mit anderen Worten: Die deutlich größere Varianz der vokalischen Perzeptionen deutet darauf hin, dass linguistische Laien bei vokalischen Auffälligkeiten differenzierter konzeptualisieren als bei prosodischen Auffälligkeiten. Die Beschreibungen der Merkmale ${ }^{17}$ belegen dies:

\footnotetext{
${ }^{17}$ Bei dieser Zusammenstellung handelt es sich um eine einfache Auflistung. Was genau die Befragten unter ihren Angaben verstehen, wird an dieser Stelle nicht weiter behandelt. In zukünftigen Auswertungen wird gesondert auf diesen Aspekt eingegangen.
} 


\begin{tabular}{|c|c|c|}
\hline Kategorien & Belege $(n>3)$ & Anzahl \\
\hline \multicolumn{2}{|c|}{ Konsonantische Assoziationen } & 73 \\
\hline & "rollendes r" & 25 \\
\hline & "betontes r" & 16 \\
\hline & "sch-Laute" & 15 \\
\hline & "sprechen mehr so [x]" & 11 \\
\hline & 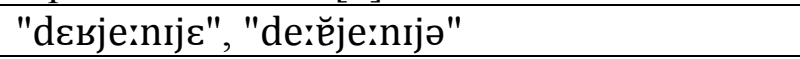 & 6 \\
\hline \multicolumn{2}{|c|}{ Vokalische Assoziation } & 23 \\
\hline & "ins ö gezogen", "o als ö" & 9 \\
\hline & "Jinst" (einst) & 5 \\
\hline & "notvint", "noِ:tvint" (Nordwind) & 5 \\
\hline & "das i so betont" & 4 \\
\hline \multicolumn{2}{|c|}{ Prosodische Assoziation } & 40 \\
\hline & $\begin{array}{l}\text { "dieses Singende", "Sing-Sang", "am Tonfall } \\
\text { sofort erkannt", "Klang", "Sprachmelodie", } \\
\text { "melodisch" }\end{array}$ & 17 \\
\hline & "Betonung" & 15 \\
\hline & "langsam gesprochen" & 4 \\
\hline & "ruckartig, abgehackt, mit Pausen gesprochen" & 4 \\
\hline \multicolumn{2}{|c|}{ Artikulatorische Assoziation } & 20 \\
\hline \multirow{3}{*}{ 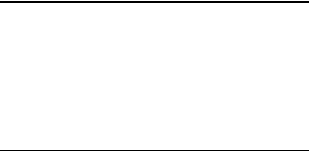 } & "undeutlich", "genuschelt" & 7 \\
\hline & "im Hals gesprochen", "kehlkopfartig" & 7 \\
\hline & "sprechen so nasal" & 6 \\
\hline
\end{tabular}

Tabelle 50: Belege für die von den 42 Befragten genannten perzipierten Merkmale (n > 3).

Unter den Merkmalen der konsonantischen Assoziation weist mit 25 Nennungen die apikale Realisierung des [r], mit der v. a. das Sprachraumkonzept Bairisch aktiviert wurde, die größte Auffälligkeit auf. Die übrigen, meist sehr allgemein beschriebenen auffälligen RRealisierungen konnten nicht mit der Aktivierung eines bestimmten Sprachraumkonzepts in einen Zusammenhang gebracht werden. Dies trifft ebenso für die postalveolare Realisierung zum Frikativ [ $[$ ] zu wie bspw. in $<$ sich $>$ wie auch für die Spirantisierung des velaren Plosivs in $<$ derjenige $>$.

Bei den vokalischen Merkmalen stach die Zentralisierung von [o] zu [ө] im Zusammenhang mit dem Konzept Ostdeutsch hervor. Mit der Rundung des Diphthongs [al] in <einst> wird bei einigen Befragten das Konzept Bairisch aktiviert, das Norddeutschkonzept hingegen mit der Vokalisierung und Dehnung in $<$ Nordwind $>$. Leichte Salienzeffekte zeichnen sich beim Vokal [i] ab, wobei hier leider nicht eindeutig zu rekonstruieren war, in welchen Wörtern bzw. in welcher lautlichen Umgebung dies zutraf. Gleichzeitig wurden eine Reihe von Einzelnennungen durch Imitation erzeugt, in denen ein gedehntes [i] auffiel, z. B. [ftвi:t n] (stritten).

Die prosodischen Merkmale sind sehr unspezifisch und allgemein gefasst und weisen - vor dem Hintergrund, dass es sich um einen vorgelesenen Text handelt - keine ausgeprägte Triggerfunktion auf, sondern werden eher zur Begründung der regionalen Identifikation herangezogen. Die Intonation wird dann vor allem als "singend" beschrieben, daneben werden unspezifische Auffälligkeiten des Tonfalls, des Klangs oder der Sprachmelodie, diverse Auffälligkeiten der Betonung sowie Aussagen zur Quantität der Sprechweise ("ruckartig" usw.) wahrgenommen.

Dasselbe trifft auch zu für die Merkmale der artikulatorischen Assoziation, hier wurde die Sprechweise insgesamt sehr unspezifisch beschrieben. 
Welche lautlichen Merkmale, die im Verdacht stehen, als Triggermerkmale für die Verortung der Sprechproben zu fungieren, können nun als salient angenommen werden? Die folgende Abbildung stellt noch einmal die perzipierten Merkmale dar, anhand derer die Befragten die Samples verortet haben. Dabei entsprechen die rot markierten Hervorhebungen den vokalischen und die grün markierten den konsonantischen Merkmalen.

Einst stritten sich Nordwind und Sonne, wer von innen beiden wohl der

Stärkere wäre, als ein Wanderer, der in einen warmen Mantel gehüllt war, des

Weges daherkam. Sie wurden einig, dass derjenige für den Stärkeren gelten

sollte, der den Wanderer zwingen würde, seinen Mantel abzunehmen. [...]

Abbildung 3: Vokalische \& konsonantische Merkmale.

Die aufgeführten Merkmale innerhalb der Abbildungen werden dabei sowohl unabhängig von der Häufigkeit ihrer Nennungen als auch unabhängig vom jeweils zugesprochenen Ort dargestellt.

Einen kleinen Vorgeschmack auf das Potenzial zukünftiger Auswertungen zu perzipierten lautlichen Merkmalen bei der lokalen Identifikation von regionalen Sprechweisen soll das Beispiel des Wortes Wanderer geben:

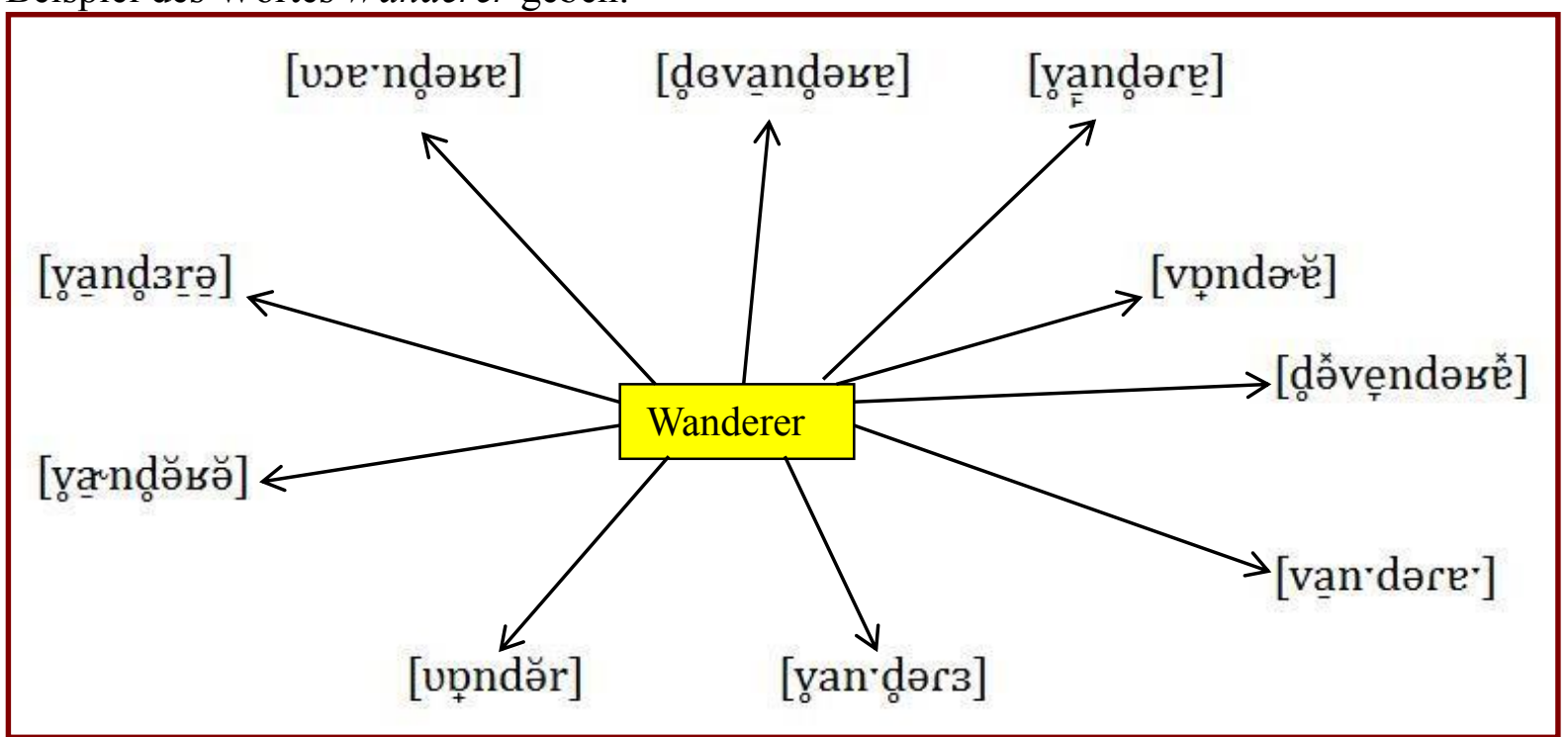

Abbildung 4: Beispiel Wanderer.

Anhand der hier dargestellten Transkriptionen der von den Gewährspersonen reproduzierten Auffälligkeiten können saliente perzipierte Merkmale herausgearbeitet und mit den Dialektkonzepten, die damit von den Gewährspersonen in Verbindung gebracht werden, abgeglichen werden. Die auf diese Weise extrahierten perzipierten Merkmale könnten dann mit anderen erhobenen Variablen wie bspw. "Gefallen" und "Korrektheit", biografischen und demografischen Variablen oder auch Sprachnormvorstellungen verglichen werden. Neben vokalischen und konsonantischen Perzeptionen kommen in der Verortung der Sprachproben vor allem auch assoziative Beurteilungen zum Tragen. Anders (2010: 269) hat in ihrem Kategoriensystem hierfür 6 Gruppen mit insgesamt 15 Subkategorien vorgesehen.

Anhand des bereits vorgestellten Datenmaterials soll nun gezeigt werden, welche Mittel außerhalb der phonetischen, artikulatorischen und prosodischen Ebene linguistische Laien zur Verfügung haben, dargebotene Sprachproben zu beschreiben. Der Fokus wird hierbei weniger 
auf der Beschreibung einzelner Sprachproben liegen. Vielmehr wird ein Überblick zu den Gewichtungen der Kategorien anhand einzelner Belege aus dem Korpus geboten.

\begin{tabular}{|c|c|c|}
\hline Gruppen & Kategorien & Anzahl \\
\hline \multicolumn{2}{|c|}{ Wortassoziationen: inhaltsbezogen } & 1 \\
\hline & Allgemeine lexikalische Merkmale & 0 \\
\hline & Lexikalische Besonderheiten & 0 \\
\hline & Phraseologisches (kulturelle Schibboleths) & 1 \\
\hline \multicolumn{2}{|c|}{ Wortassoziationen: ausdrucksbezogen } & 51 \\
\hline & Wörter/Wortgruppen als phonetische Konglomerate & 51 \\
\hline \multicolumn{2}{|c|}{ Dialektbeschreibung } & 153 \\
\hline & Allgemeine Beschreibungen & 21 \\
\hline & Qualifizierende Beschreibungen & 29 \\
\hline & Relationale Beschreibungen & 35 \\
\hline & Beschreibungen mit Identifikationscharakter & 50 \\
\hline & $\begin{array}{l}\text { Personen-/Gruppenbeschreibungen mit } \\
\text { Verweisungsfunktion auf lautliche Besonderheiten }\end{array}$ & 18 \\
\hline \multicolumn{2}{|c|}{ Dialektbewertung } & 49 \\
\hline & Allgemeine Bewertungsebene & 32 \\
\hline & Evaluative Bewertungsebene & 17 \\
\hline \multicolumn{2}{|l|}{ Variation } & 89 \\
\hline & Vertikale Variation (Standard-Substandard) & 37 \\
\hline & $\begin{array}{l}\text { Horizontale Variation (Dialektbezeichnungen, } \\
\text { Grenzbereiche, Übergänge }\end{array}$ & 52 \\
\hline \multicolumn{2}{|c|}{ Raumparameter } & 58 \\
\hline & Geografische/politische Orientierung & 55 \\
\hline & Räumliche Orientierung & 3 \\
\hline
\end{tabular}

Tabelle 61: Außerphonetische Kategorien und die Anzahl ihrer Belege.

Die Tabelle zeigt zuerst, dass im Datenmaterial mit einer Ausnahme keine inhaltsbezogenen Wortassoziationen vorliegen. Die Gewährspersonen haben weder lexikalische Merkmale noch auffällige Besonderheiten auf lexikalischer Ebene perzipiert. Dieses Ergebnis ist aber nicht weiter verwunderlich, da es sich bei den Sprachbeispielen um Vorlesetexte handelt. Der einzige Beleg innerhalb dieser Gruppe von einer Gewährsperson aus Hameln, die äußerte, "ein typisches Wort" in der Hochalemannischen Sprechprobe erkannt zu haben (Original-Beleg: "weil da war gerade wieder irgendwo ein wort dass typisch sozusagen"). Allerdings konnte sie dieses Wort auf Nachfrage nicht benennen.

Auf der Ausdrucksseite ließen sich hingegen 51 Belege feststellen, die sich auf Wörter und Wortgruppen beziehen, an denen eine Sprachprobe vermeintlich erkannt wurde. Innerhalb dieser Gruppe können zwei Arten von Belegen bestimmt werden. Die eine beinhaltet offenbar Lexeme, die anhand vokalischer Auffälligkeiten perzipiert und wiederholt wurden (z. B. "als ein wonderer", "'beiden' die betonen das so"), die andere Gruppe beinhaltet Wörter, die durch ihre Konsonanten auffällig erscheinen (z. B. "'stritten sich' oder wie also das fand ich war sehr markant", "weiß nicht ob er jetzt 'isch' gesagt hat aber so klang es"). Auffällig war hier vor allem die Brandenburgische Sprachprobe, in der vor allem "derjenige", vom Potsdamer Sprecher mit spirantischem [g] realisiert, perzipiert wurde. Nicht immer führten die auffälligen Lexeme zur korrekten Verortung der Sprechprobe. Oft wurde den Wörtern zwar eine gewisse Auffälligkeit bescheinigt, jedoch waren einige Gewährspersonen dann nicht in der Lage, diese Auffälligkeit mit den dargebotenen Städten in Verbindung zu bringen. 
In der Gruppe der dialektbeschreibenden Äußerungen sind mit insgesamt 153 Eintragungen die meisten Belege zu finden. Die Kategorie "Beschreibungen mit Identifikationscharakter" kann 50 Belege aufweisen und ist damit die zahlenmäßig größte. Die Inhalte beziehen sich in erster Linie auf perzipierte Merkmale innerhalb der Sprachprobe, die den Gewährspersonen helfen, das Sprachmaterial korrekt zuzuordnen. Typischerweise fallen hierunter Äußerungen wie: "österreichischer touch", "das hat mich so ein bisschen an das östliche erinnert". Wenn keine identifizierenden Merkmale perzipiert wurden, haben viele Gewährspersonen versucht, eine Relation zwischen dem gerade Gehörten und einer anderen Sprechweise herzustellen, sodass die Kategorie "Relationale Beschreibungen" diejenige mit der zweitgrößten Belegdichte darstellt. Häufig wurden hier vorangegangene Sprachproben als Vergleichsmuster herangezogen und an ihnen die Eigenheiten der aktuellen Sprachprobe erläutert, oder es wurde, wie im Interviewleitfaden vorgegeben, die aktuelle Sprachprobe mit der standardnahen Kontrollvarietät abgeglichen (z. B. "unterscheidet sich deutlich von der vorherigen [Helmstedt, S. Sch.]", "bisschen singsang wie im sächsischen"). In dieser Gruppe kommt teilweise eine Schwäche des Settings zu tragen, denn durch die randomisierte Reihenfolge der zur Auswahl stehenden Städte kommt es bei der Zuordnung durch die Probanden häufig zu einem Ausschlussverfahren. Dies passiert insbesondere bei einer großen Entfernung zwischen den Städten untereinander.

Interessant ist außerdem die Kategorie "Personen- und Gruppenbeschreibungen mit Verweisfunktion auf lautliche Besonderheiten", die mit 18 Belegen darauf hinweist, dass die Sprachproben offenbar keine Assoziationen zu bestimmten Personen hervorruft. Trotzdem lassen sich innerhalb der wenigen Belege zwei Arten von Äußerungen der Gewährspersonen bestimmen. Einerseits werden Verwandte und Bekannte als Beispiele herangezogen, andererseits bedienen sich die Probanden öffentlicher Personen, um die Verortung der Sprechprobe zu erklären (z. B. "nein also wie gesagt horst seehofer würde mir jetzt spontan einfallen der spricht auch immer so"). ${ }^{18}$ Eine Gewährsperson aus Schleiden konnte diesen Effekt sogar verbalisieren: "ich kann das vielleicht auch so durch die menschen die man getroffen hat dass man das so ein bisschen damit verbindet".

In den "Qualifizierenden Beschreibungen" sind vor allem Äußerungen enthalten, die die Typizität aus subjektiver Sicht der Gewährspersonen beschreiben. Diese kamen vor allem bei der Verortung der Wienerischen Sprechprobe zum Ausdruck (z. B. "typisch Wienerisch"). Interessanterweise war die Zentralhessisch/Rheinfränkische Sprachprobe diejenige, bei der keine Typizität festgestellt werden konnte. Es scheint hier einen Zusammenhang zur Zuordnungsrichtigkeit zu geben, bei der die Sprachprobe aus Darmstadt einen der hinteren Plätze belegt, also oft falsch zugeordnet wurde (s. o.).

Die Bewertung der Sprechproben (Gruppe "Dialektbewertung") findet hauptsächlich auf allgemeiner Ebene statt. Es werden vor allem solche Eigenschaften bewertet, die die Gewährspersonen zu den einzelnen Sprechproben erwarten. Hierbei spielt die Intensität dieser Eigenschaften die Hauptrolle, wie die folgenden Beispiele zeigen: "der charme hat noch ein bisschen gefehlt" (Gewährsperson aus Vaduz zur Wiener Sprechprobe), "zu lang gezogen" (Gewährsperson aus Simmern zur Wiener Sprechprobe). Auf evaluativer Ebene werden besonders die Sprechproben aus dem süddeutschen Sprachgebiet (Wien, Traunstein, Zürich, Darmstadt) bewertet und häufig mit "schlechter Sprache" in Verbindung gebracht. So äußert sich bspw. eine Gewährsperson aus Schleiden zur obersächsischen Sprechprobe wie folgt: "war nicht gut sächsisch also". Die mögliche These, das Obersächsische werde besonders schlecht konnotiert, da es zu den unbeliebteren Dialekten gehöre (cf. Hundt 2010: 179-219) konnte in diesem Sample nicht bestätigt werden; die Anzahl an evaluativen Äußerungen ist gering -

${ }^{18}$ Eine "Prominentenfrage", also die Frage, wer aus dem öffentlichen Leben ähnlich spricht, ist in der Befragung für die Makrokartierung vorgesehen. 
dennoch liegen die Gefallen-Werte für das obersächsische Sprachbeispiel überwiegend im Negativbereich. ${ }^{19}$ Insgesamt beziehen sich die Bewertungen generell weniger auf die Sprechweise im Allgemeinen als vielmehr auf die in dem Moment abgespielte Sprachprobe.

Eine Bewertung der Sprechproben erfolgt meistens auf Nachfrage durch die Exploratorin. Bei der ersten Verortung hingegen spielen die Kategorien "Variation" und "Raumparameter" die entscheidenden Rollen, wobei sie sich häufig gegenseitig ausschließen: Wenn die Gewährsperson die Sprachprobe erkennt und ihr ein Konzept zuordnen kann (z. B. Schwäbisch), erfolgen nur selten noch weitere Erläuterungen zur räumlichen oder geografischen Einordnung. Diese erfolgen allerdings, wenn der Gewährsperson ein für sie passendes Konzept fehlt, wie z. B. bei der Verortung der Zentralhessisch/Rheinfränkischen Sprachprobe. Die Probanden bringen die Sprachprobe nicht mit Darmstadt in Verbindung, sodass geografische Parameter zur Verortung hinzugezogen werden. In diesem Fall wird die Sprechweise häufig in den südlichen Raum verortet, wohingegen die Brandenburgische, Nordniederdeutsche und Ostfälische Sprachprobe - häufig mit der Aussage "das war hochdeutsch/dialektfrei/ohne dialekt" versehen - tendenziell in den Norden verortet werden.

Insgesamt offenbart die Untersuchung der Sprachprobenverortung mit Hilfe des Kategoriensystems einen interessanten Einblick in die Möglichkeiten linguistischer Laien, Sprachbeispiele zu verorten, zu bewerten und zu beschreiben. Dabei fällt insbesondere die Anzahl und Varianz der Beschreibungen auf. Diese hängen häufig mit außersprachlichen Parametern, wie Personen aus dem eigenen Umfeld, bekannten Persönlichkeiten, aber auch Stereotypen zusammen. In weiteren Forschungsarbeiten wäre es fruchtbringend, wenn die Verortung einzelner Sprachproben untersucht würde. So zeigte sich bereits in dieser Untersuchung, dass bspw. das Zentralhessisch/Rheinfränkische äußerst unterschiedlich perzipiert und kommentiert wird, da von einer Darmstädter (also: hessischen) Sprachprobe offensichtlich etwas anderes erwartet wird. Die Frage nach dem laienlinguistisch "typisch" Hessischen, der objektlinguistischen Wirklichkeit und den Konvergenzen bzw. Diskrepanzen dazwischen, wäre ein sicherlich lohnender Untersuchungsgegenstand.

\section{$5 \quad$ Fazit und Ausblick}

Das für die Untersuchung konzipierte Ratespiel offenbart eine Vielzahl an Möglichkeiten, das Repertoire linguistischer Laien zur Konzeptualisierung von Sprachräumen aufzudecken und zu analysieren. Zum einen können ganz allgemeine/quantitative Bewertungen zu den Sprechproben erhoben werden (cf. Gefallen und Korrektheit Tab. 5 u. 6), die einen ersten Einblick geben. Zum anderen bieten die weiteren Fragestellungen innerhalb des Ratespiels Raum für weitere Analysen der assoziierten und perzipierten Merkmale.

Auch wenn - wie am Beispiel "Wanderer" angedeutet - einzelne Wörter ein ausgeprägteres Konzeptualisierungspotenzial aufweisen als andere, fällt dennoch innerhalb der Auswertung der Daten auf, dass auf die erste Frage "Woran haben Sie die Herkunft des Sprechers erkannt?" insgesamt nur wenige Antworten zu verzeichnen sind. Auffällig ist, dass erst im späteren Gesprächsverlauf Begründungen oder auch detaillierte Angaben zu den sprachlichen Merkmalen der Sprechprobe geäußert werden. Somit wird häufig erst im zweiten Anlauf auf stereotype Vorstellungen und Generalisierungen zurückgegriffen.

Festhalten lässt sich erstens, dass auf einen Sprachraum bezogene konzeptspezifische Merkmale der lautlichen Besonderheiten nur bei den vokalischen und konsonantischen Merkmalen, nicht jedoch bei den prosodischen und artikulatorischen Merkmalen auftreten.

\footnotetext{
19 Diese Tatsache könnte der Interviewsituation geschuldet sein. Es stellte sich während der Befragungen heraus, dass sich viele Probanden scheuen, Dialekte und Sprechweisen zu bewerten, wenn sie dazu aufgefordert wurden (z. B. Frage 4.1.3 aus dem Interviewleitfaden: "Wie gefällt Ihnen die Sprechweise auf einer Skala von -3 bis $+3 ")$.
} 
Zweitens scheinen die Sprachraumkonzepte "Bairisch/Süddeutsch", "Schweizerdeutsch", "Ostdeutsch" und "Norddeutsch" anfälliger zu sein für die lokale Identifikation regional gefärbter Standardsprache als andere Sprachraumkonzepte. Drittens zeigte sich, dass viele Befragte einen "ostdeutschen", nicht jedoch einen "westdeutschen" Sprachraum konzeptualisieren.

Außerdem konnte festgestellt werden, dass die Begründungen vom Typ Ausschlussprinzip ausführlicher betrachtet und analysiert werden müssen, denn die Gewährsperson kann zwar nicht begründen, warum der Sprecher aus dem jeweiligen Ort stammen muss, er kann aber genau begründen, warum er NICHT aus einem anderen Ort kommen kann. Ein angemerkter Kritikpunkt der Interviewten ist ebenfalls, dass sie die Sprechproben nicht beliebig oft hören können, um sich besser an die unmittelbar perzipierten Merkmale erinnern zu können. Dieser Umstand ist auch im Untertitel dieses Beitrags angedeutet: "in dem moment wo ich es dann erkenne dann ist es auch gleich wieder weg".

Die Untersuchung der Kategorien, die sich mit assoziativen/perzipierten Beschreibungen, Bewertungen und Beurteilungen hinsichtlich der Variation und den Raumparametern beschäftigen, ergab, dass es sich hierbei um eine wichtige Variable in der Rekodierung von Sprachkonzepten linguistischer Laien handelt. Sie weist insbesondere in der Beschreibung der vorgespielten Sprachproben eine große Varianz auf und eröffnet der Forschung einen interessanten Einblick in das Repertoire, das den Gewährspersonen zur Beschreibung von Sprachraumkonzepten zur Verfügung steht.

In den weiteren Analyseschritten werden die Daten zum einen nach den Kriterien Tokenfrequenz sowie konzeptspezifische saliente Merkmale ausführlich ausgewertet. Auf Phänomene des indirekten Sprachwissens, wie sie durch Imitationen hervorgerufen werden können (cf. Purschke 2012) soll zum anderen in der weiteren Auswertung des Kieler DFGProjekts eingegangen werden, um letztlich das Auftreten und die Funktion von Salienz präziser fassen und beschreiben zu können.

\section{Literatur}

Allensbacher Berichte (2008): Auch außerhalb von Bayern wird Bayerisch gern gehört. (= Allensbacher Berichte 4=). http://www.ifd-allensbach.de/uploads/tx_reportsndocs/ prd_0804.pdf, letzter Zugriff am 21.04.2014.

Anders, Christina A. (2010): Wahrnehmungsdialektologie. Das Obersächsische im Alltagsverständnis von Laien. Berlin/New York: de Gruyter.

Auer, Peter/Barden, Birgit/Großkopf, Beate (1996): "Dialektanpassung bei sächsischen

"Übersiedlern" - Ergebnisse einer Longitudinalstudie". In: Boretzky, Norbert/Enninger, Werner/Stolz, Thomas (eds.): Areale, Kontakte, Dialekte. Sprache und ihre Dynamik in mehrsprachigen Situationen. Bochum, Brockmeyer: 139-166.

Der deutsche Sprachraum aus der Sicht linguistischer Laien. http://www.wahrnehmungs dialektologie.uni-kiel.de/, letzter Zugriff am 21.04.2014.

Elmentaler, Michael/Gessinger, Joachim/Wirrer, Jan (2010): "Qualitative und quantitative Verfahren in der Ethnodialektologie am Beispiel von Salienz". In: Anders, Christina A./Hundt, Markus/Lasch, Alexander (eds.): Perceptual dialectology. Neue Wege der Dialektologie. Berlin/New York, de Gruyter: 111-149. (=Linguistik-Impulse - Tendenzen 38).

Elmentaler, Michael (2012): "In Hannover wird das beste Hochdeutsch gesprochen". In: Anderwald, Lieselotte (ed.): Sprachmythen - Fiktion oder Wirklichkeit? Frankfurt a. M. etc., Lang: 101-115.

Forschungszentrum Deutscher Sprachatlas (ed.): Regionalsprache.de. http://www.regional sprache.de/, letzter Zugriff am 21.04.2014. 
Herrgen, Joachim/Schmidt Jürgen Erich (1985): "Systemkontrast und Hörerurteil. Zwei Dialektalitätsbegriffe und die ihnen entsprechenden Messverfahren". Zeitschrift für Dialektologie und Linguistik 52: 20-42.

Hundt, Markus (1992): Einstellung gegenüber dialektal gefärbter Standardsprache. Eine empirische Untersuchung zum Bairischen, Hamburgischen, Pfälzischen und Schwäbischen. Stuttgart: Steiner.

Hundt, Markus (2010): "Bericht über die Pilotstudie "Laienlinguistische Konzeptionen deutscher Dialekte"". In: Anders, Christina A./Hundt, Markus/Lasch, Alexander (eds.): Perceptual dialectology. Neue Wege der Dialektologie. Berlin/New York, de Gruyter: 179219. (= Linguistik - Impulse - Tendenzen 38).

Institut für Deutsche Sprache (ed.): Hör mal, wo der spricht. http://multimedia.idsmannheim.de/hoermal/web/, letzter Zugriff am 21.04.2014.

Kehrein, Roland (2012a): Regionalsprachliche Spektren im Raum-Zur linguistischen Struktur der Vertikale. Stuttgart: Steiner. (= Zeitschrift für Dialektologie und Linguistik Beihefte 152).

Kehrein, Roland (2012b): "Wen man nicht alles für einen Sachsen hält?! Oder: Zur Aktivierung von Sprachraumkonzepten durch Vorleseaussprache". In: Hünecke, Rainer/Jakob, Karlheinz (eds.): Die obersächsische Sprachlandschaft in Geschichte und Gegenwart. Heidelberg, Winter: 223-263.

Kerswill, Paul/Williams, Ann (2002): "'Salience" as an Explanatory Factor in Language Change. Evidence from Dialect Levelling in Urban England". In: Jones, Mari C./Esch, Edith (eds.): Language Change. The Interplay of Internal, External and Extra-Linguistic Factors. Berlin/New York, de Gruyter: 81-110.

Kleiner, Stefan (ed.): Das Korpus "Deutsch heute" im Projekt "Variation des gesprochenen Deutsch". http://www1.ids-mannheim.de/prag/AusVar/Deutsch_heute, letzter Zugriff am 21.04.2014.

Lenz, Alexandra N. (2003): Struktur und Dynamik des Substandards. Eine Studie zum Westmitteldeutschen (Wittlich/Eifel). Stuttgart: Steiner.

Lenz, Alexandra N. (2010): "Zum Salienzbegriff und zum Nachweis salienter Merkmale". In: Anders, Christina A./Hundt, Markus/Lasch, Alexander (eds.): Perceptual dialectology. Neue Wege der Dialektologie. Berlin/New York, de Gruyter: 89-110. (= Linguistik Impulse-Tendenzen 38).

Mattheier, Klaus J. (1996): "Varietätenkonvergenz. Überlegungen zu einem Baustein einer Theorie der Sprachvariation". In: Auer, Peter et al. (eds.): Konvergenz und Divergenz von Dialekten in Europa. Tübingen, Niemeyer: 31-52. (= Sociolinguistica 10).

Mihm, Arend (1985): "Prestige und Stigma des Substandards. Zur Bewertung des Ruhrdeutschen im Ruhrgebiet". In: Mihm, Arend (ed.): Sprache an Rhein und Ruhr. Stuttgart, Steiner: 163-193.

Preston, Dennis R. (1999): Handbook of Perceptual Dialectology. Band 1. Amsterdam/Philadelphia: John Benjamins.

Purschke, Christoph (2011): Regionalsprache und Hörerurteil. Grundzüge einer perzeptiven Variationslinguistik. Stuttgart: Steiner.

Schirmunski, Viktor M. (1928/1929): "Die schwäbischen Mundarten in Transkaukasien und Südukraine". Teuthonista 5: 3860, 157-171.

Schirmunski, Viktor M. (1930): "Sprachgeschichte und Siedlungsmundarten". In: GermanischRomanische Monatsschrift 18: 113-122, 171-188.

Schmidt, Jürgen Erich/Herrgen, Joachim (2011): Sprachdynamik. Eine Einführung in die moderne Regionalsprachenforschung. Berlin: Erich Schmidt Verlag.

Trudgill, Peter (1986): Dialects in contact. Oxford: Blackwell.

Wirrer, Jan (ed.): Sprachvariation in Norddeutschland. http://www.corpora.unihamburg.de/sin/index.html, letzter Zugriff am 21.04.2014. 